\title{
Internal redundancy: an approach to improve the dynamic parameters around sharp corners
}

\author{
S. S. Parsa ${ }^{1}$, J. A. Carretero ${ }^{1}$, and R. Boudreau ${ }^{2}$ \\ ${ }^{1}$ Department of Mechanical Engineering, University of New Brunswick, Fredericton, NB, Canada \\ ${ }^{2}$ Département de génie mécanique, Université de Moncton, Moncton, NB, Canada
}

Correspondence to: J. A. Carretero (juan.carretero@unb.ca)

Received: 11 December 2012 - Revised: 8 March 2013 - Accepted: 5 April 2013 - Published: 28 May 2013

\begin{abstract}
In recent years, redundancy in parallel manipulators has been studied broadly due to its capability of overcoming some of the drawbacks of parallel manipulators including small workspaces and singular configurations. Internal redundancy, first introduced for serial manipulators, refers to the concept of adding movable masses to some links so as to allow to control the location of the centre of mass and other dynamic properties of some links. This concept has also been referred to as variable geometry. This paper investigates the effects of internal redundancy on the dynamic properties of a planar parallel manipulator while performing a family of trajectories. More specifically, the 3-RRR planar manipulator, where a movable mass has been added to the distal link, is allowed to trace trajectories with rounded corners and different radii. The proposed method uses the manipulator's dynamic model to actively optimise the location of the redundant masses at every point along the trajectory to improve the dynamic performance of the manipulator. Numerical examples are shown to support the idea.
\end{abstract}

\section{Introduction}

Redundancy in parallel manipulators is normally divided into kinematic redundancy, actuation redundancy and branch redundancy (Lee and Kim, 1993; Zanganeh and Angeles, 1994; Merlet, 1996; Ruggiu and Carretero, 2009; Boudreau and Nokleby, 2012). Actuation redundancy consists of replacing passive joints with active ones (Zanganeh and Angeles, 1994; Cheng et al., 2003, 2011; Nokleby et al., 2005) where the number of degrees-of-freedom or mobility of the manipulator does not change. Although actuation redundancy can help either eliminate or reduce singular configurations, issues such as force interference make the manipulators more complex to analyze, design and control (Firmani and Podhorodeski, 2004; Garg et al., 2009). The second type of redundancy is called branch redundancy where an extra actuated branch is added to the manipulator (Firmani et al., 2007). Branch redundancy can improve the force capabilities of the manipulator and reduce the number of singular configurations. The third type of redundancy is called kinematic redundancy where active joints and links are added to one or more branches of the manipulator (Merlet, 1996; Wang and Gosselin, 2004). This type of redundancy can enhance the dexterity of the manipulator as well as enlarge the workspace. Additionally, kinematic redundancy allows to plan trajectories far from certain singular configurations as the inverse displacement problem has an infinite number of solutions (thus often allowing for the manipulator to remain as far as possible from singular configurations) (Ebrahimi et al., 2008).

Redundant parallel manipulators have been widely used to improve the trajectories of parallel robots. For instance, Cha et al. (2007) showed that kinematically redundant manipulators can effectively avoid singular configurations thus increasing the singularity-free workspace of the parallel manipulator.

Ruggiu and Carretero (2010) applied an optimisation procedure on a kinematically redundant parallel manipulator to minimise the acceleration of the actuators while following certain trajectories. The method was applied on a kinematically redundant parallel manipulator following square paths with rounded corners. They showed that the accelerations of 


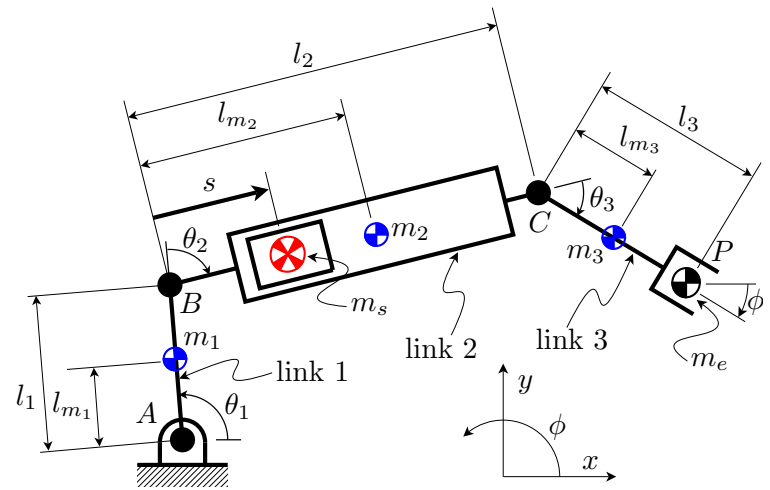

Figure 1. Serial manipulator with internal redundancy in link 2. The location of mass $m_{s}$ can be changed without altering the position of the end effector (point $p$ ).

the actuated joints on the kinematically redundant manipulator are significantly less than the ones needed for a nonredundant manipulator.

Recently, a new type of redundancy called internal redundancy has been the focus of some attention in the context of serial manipulators (Vukobratović et al., 2000). Similar to the types of redundancy described earlier, a new set of degrees of freedom (DOF) is added to the serial manipulator. However, in contrast with the redundant actuators and/or links described earlier, the new DOF is used to change the internal geometry of a link resulting in the change of the location of the link's centre of mass and its inertial mass distribution parameters (i.e., its mass moment of inertia). Since the changes are made within the internal members of the link, the redundant DOF does not have a direct effect on the end effector pose (i.e., position and orientation). More specifically, in reference to Fig. 1, the position of the mass $m_{s}$ in link 2 can be changed without altering the pose of the end effector. This allows for different internal motions for a given trajectory of the end effector thus adding control to some dynamic parameters of the manipulator to attempt to improve its dynamic performance for specific tasks.

In this paper, the concept of internal redundancy is applied to a planar parallel manipulator. First, a 3-RRR manipulator with internal redundancy in all three branches is described and its kinematic and dynamic equations (Sects. 2 and 3) are derived. Then, an optimisation problem is formulated where the displacement of each of the portable masses at every point throughout a trajectory is sought to minimise the torques at the base actuators (Sect. 4). The architectural parameters and trajectory planning algorithm are explained through a numerical example and are presented in Sect. 5 and then discussed in more detail in Sect. 6. Finally, Sect. 7 presents the conclusions and briefly discusses potential future work.

\section{The 3-RRR manipulator with internal redundancy}

A 3-DOF planar parallel manipulator shown in Fig. 2a is chosen to investigate the effect of internal redundancy in parallel manipulators. The manipulator is a symmetrical 3-RRR manipulator with base $\left(G_{1} G_{2} G_{3}\right)$ and end effector $\left(A_{1} A_{2} A_{3}\right)$ as equilateral triangles. The three revolute actuators to move the manipulator's end effector are located at $G_{i}$, the base joint of each branch. The length of the proximal links, i.e., links $G_{i} B_{i}$ $(i=1,2,3)$, has been denoted by $l_{1}$ while the length of the distal link, i.e., $B_{i} A_{i}(i=1,2,3)$ has been denoted by $l_{2}$.

In order to study the concept of internal redundancy, a portion of the distal link (the portion from $B_{i}$ to $A_{i}^{\prime}$ ) protrudes on the opposite side of the revolute joint at $B_{i}$ and creates a linear track from $A_{i}$ to $A_{i}^{\prime}$ where the redundant mass $m_{s}$ can slide on (see Fig. 2b). The position of the mass relative to the elbow joint $B_{i}$ is given by $s_{i}$ and is measured in the direction of $A_{i}$. Since the masses $m_{s}$ are mounted on tracks or prismatic joints, their position along $A_{i} A_{i}^{\prime}$ can be actively controlled. More specifically, the distance $s_{i}$ from elbow joint $B_{i}$ to the centre of mass $m_{s}$ can be actively controlled thus changing the overall dynamic properties and effects of links $A_{i}^{\prime} A_{i}$.

To help complete the dynamic model, each element has been given a mass while symmetry has been assumed to simplify the analysis. Moreover, the links have been modelled as slender rods. The proximal links have all been assigned a mass $m_{1}$ with their centre of mass located halfway between $G_{i}$ and $B_{i}$ while all three distal links have been assigned a mass $m_{2}$ with their centre of mass located halfway between $A_{i}^{\prime}$ and $A_{i}$. The moving platform has been assigned a mass $m_{e}$ with its barycentre located at the centroid of the moving platform.

\section{Dynamic model of the redundant 3-RRR manipulator}

The inverse dynamic problem of a 3-RRR planar parallel manipulator with 3-DOF of internal redundancy is developed in this section. The dynamic model is obtained using the Principle of Virtual Work as well as d'Alembert's principle. The derivation is similar to that presented in Wu et al. (2011). For this purpose, a complete kinematic model of the manipulator needs to be developed to derive the velocity and acceleration equations. In addition to that, inertial forces and moments of all the links need to be calculated.

Note that in what follows, the equations are derived for each of the three legs. Therefore, index $i$ in the equations that follow is assumed to respectively take the values 1,2 and 3 when deriving the equations for legs 1,2 and 3.

\subsection{Kinematics}

The base coordinate frame $O-x y$ (denoted by $\{O\}$ ) shown in Fig. 2 is fixed on point $G_{1}$. Also, a moving coordinate frame $P-x_{N} y_{N}$ (denoted by $\{N\}$ ) is attached to the barycentre of the 

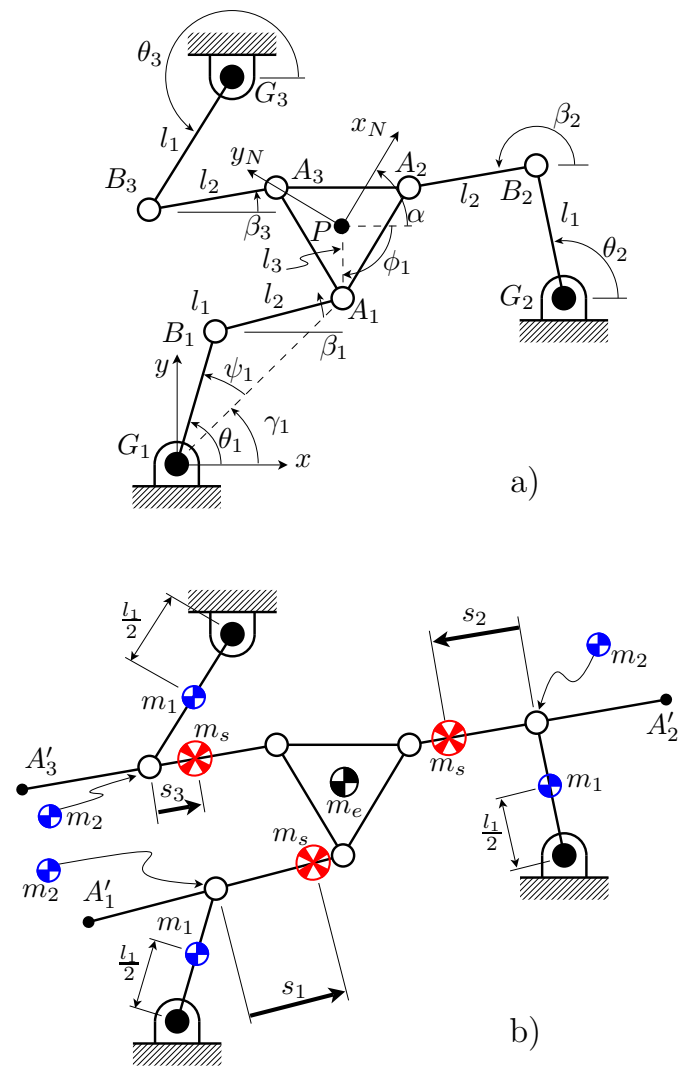

Figure 2. 3-RRR planar manipulator: (a) basic kinematic parameters and (b) location of the centre of mass of each component $\left(l_{i}\right.$ are fixed values while $s_{i}$ are variable).

moving platform. The position vector of $B_{i}$ in the base coordinate frame is defined as follows:

$\mathbf{r}_{B_{i}}=\mathbf{r}_{G_{i}}+l_{1}\left[\begin{array}{c}\cos \theta_{i} \\ \sin \theta_{i}\end{array}\right]$

where $\mathbf{r}_{B_{i}}$ describes the position vector of point $B_{i}, \mathbf{r}_{G_{i}}$ is the position vector of point $G_{i}$ and $\theta_{i}$ is the angle link $G_{i} B_{i}$ makes with the x-axis (i.e., the actuation variable for the motor located at $G_{i}$ ). The position vector of $A_{i}$ is expressed as follows:

$\mathbf{r}_{A_{i}}=\mathbf{r}_{B_{i}}+l_{2}\left[\begin{array}{c}\cos \beta_{i} \\ \sin \beta_{i}\end{array}\right]=\mathbf{r}_{p}+\mathbf{R r}_{A_{i}}^{N}$

where $\mathbf{r}_{A_{i}}$ is the position vector of point $A_{i}, \beta_{i}$ describes the angle of link $B_{i} A_{i}$ with respect to the horizontal $x$ direction, $\mathbf{r}_{p}$ is the position vector of point $p$ and $\mathbf{r}_{A_{i}}^{N}$ is the position vector of $A_{i}$ expressed in frame $N$. The rotation matrix $\mathbf{R}$ describing frame $\{N\}$ relative to frame $\{O\}$ is defined as follows:

$\mathbf{R}=\left[\begin{array}{cc}\cos \alpha & -\sin \alpha \\ \sin \alpha & \cos \alpha\end{array}\right]$.

The constraint equation of motion is written as follows:

$\left\|\mathbf{r}_{A_{i}}-\mathbf{r}_{B_{i}}\right\|=l_{2}$ where $l_{2}$ is the portion of the distal link from $B_{i}$ to $A_{i}$.

\subsection{Inverse displacement problem}

The inverse displacement problem of the 3-RRR planar manipulator is written as follows:

$\theta_{i}=\gamma_{i} \pm \psi_{i}$

where $\gamma_{i}$ is defined as follows:

$\gamma_{i}=\operatorname{atan} 2\left(x_{A_{i}}, y_{A_{i}}\right)$

where atan 2 is the quadrant corrected inverse tangent function, while $x_{A_{i}}$ and $y_{A_{i}}$ are the Cartesian components of the position of $A_{i}$ relative to $G_{i}$ and are written as follows:

$x_{A_{i}}=x-l_{3} \cos \phi_{i}-x_{G_{i}}$

$y_{A_{i}}=y-l_{3} \sin \phi_{i}-y_{G_{i}}$

where $x$ and $y$ are Cartesian positions of point $P, l_{3}$ is the radius of the moving platform (i.e., the distance between $P$ and $A_{i}$ ) and $\phi_{i}$ is given by

$\phi_{1}=\alpha+\frac{\pi}{6} \quad \phi_{2}=\alpha+\frac{5 \pi}{6} \quad \phi_{3}=\alpha-\frac{\pi}{2}$.

The equation for $\psi_{i}$ is written as follows:

$\psi_{i}=\cos ^{-1}\left(\frac{l_{1}^{2}-l_{2}^{2}+x_{A_{i}}^{2}+y_{A_{i}}^{2}}{2 l_{1}\left(x_{A_{i}}^{2}+y_{A_{i}}^{2}\right)}\right)$.

\subsection{Velocity and acceleration}

Taking the time derivative of Eq. (1) leads to

$\dot{\mathbf{r}}_{B_{i}}=l_{1} \dot{\theta}_{i}\left[\begin{array}{c}-\sin \theta_{i} \\ \cos \theta_{i}\end{array}\right]$

where $\dot{\theta}_{i}$ is the angular velocity of actuator $i$. The linear velocity of point $A_{i}$ is written as follows:

$\dot{\mathbf{r}}_{A_{i}}=\mathbf{v}_{P}+\dot{\alpha} \mathbf{N} \mathbf{R r}_{A_{i}}^{N}=\dot{\mathbf{r}}_{B_{i}}+l_{2} \dot{\beta}_{i}\left[\begin{array}{c}-\sin \beta_{i} \\ \cos \beta_{i}\end{array}\right]$

where $\mathbf{v}_{P}$ is the vector describing the linear velocity of point $P$. Matrix $\mathbf{N}$ is defined as follows:

$\mathbf{N}=\left[\begin{array}{cc}0 & -1 \\ 1 & 0\end{array}\right]$.

The angular velocity of link $B_{i} A_{i}$ is derived from Eq. (12) and is written as follows:

$\dot{\beta}_{i}=\left[\begin{array}{ll}\frac{-\sin \beta_{i}}{l_{2}} & \frac{\cos \beta_{i}}{l_{2}}\end{array}\right]\left(\dot{\mathbf{r}}_{A_{i}}-\dot{\mathbf{r}}_{B_{i}}\right)$

The linear acceleration of points $B_{i}$ and $A_{i}$ is obtained as the time derivative of Eqs. (11) and (12):

$$
\begin{aligned}
& \mathbf{a}_{B_{i}}=l_{1} \ddot{\theta}_{i}\left[\begin{array}{c}
-\sin \theta_{i} \\
\cos \theta_{i}
\end{array}\right]-l_{1} \dot{\theta}_{i}^{2}\left[\begin{array}{c}
\cos \theta_{i} \\
\sin \theta_{i}
\end{array}\right] \\
& \mathbf{a}_{A_{i}}=\mathbf{a}_{P}+\ddot{\alpha} \mathbf{N} \mathbf{R}_{A_{i}}^{N}-\dot{\alpha}^{2} \mathbf{R r}_{A_{i}}^{N}
\end{aligned}
$$


where $\mathbf{a}_{P}$ is the linear acceleration of point $P$. The time derivative of Eq. (14) leads to

$$
\begin{aligned}
\ddot{\beta}_{i}= & {\left[\frac{-\sin \beta_{i}}{l_{2}} \frac{\cos \beta_{i}}{l_{2}}\right]\left(\mathbf{a}_{A_{i}}-\mathbf{a}_{B_{i}}\right) } \\
& -\dot{\beta}\left[\frac{\cos \beta_{i}}{l_{2}} \frac{\sin \beta_{i}}{l_{2}}\right]\left(\dot{\mathbf{r}}_{A_{i}}-\dot{\mathbf{r}}_{B_{i}}\right) .
\end{aligned}
$$

In order to generate the Jacobian matrix, the time derivative of Eq. (4) yields

$\mathbf{J}=\left[\begin{array}{ccc}\frac{-a_{1}}{c_{1}} & \frac{-b_{1}}{c_{1}} & \frac{-d_{1}}{c_{1}} \\ \frac{-a_{2}}{c_{2}} & \frac{-b_{2}}{c_{2}} & \frac{-d_{2}}{c_{2}} \\ \frac{-a_{3}}{c_{3}} & \frac{-b_{3}}{c_{3}} & \frac{-d_{3}}{c_{3}}\end{array}\right]$

where the elements of this Jacobian matrix are as follows:

$$
\begin{aligned}
a_{i}= & -h_{1}\left(x-x_{G_{i}}\right)+h_{3} \cos \theta_{i}+h_{1} \cos \phi_{i} \\
b_{i}= & -h_{1}\left(y-y_{G_{i}}\right)+h_{1} \sin \theta_{i}+h_{2} \sin \phi_{i} \\
c_{i}= & h_{3}\left[\left(y-y_{G_{i}}\right) \cos \theta_{i}-\left(x-x_{G_{i}}\right) \sin \theta_{i}\right] \\
& +\sin \left(\theta_{i}-\phi_{i}\right) \\
d_{i}= & h_{2}\left[\left(y-y_{G_{i}}\right) \cos \phi_{i}-\left(x-x_{G_{i}}\right) \sin \phi_{i}\right] \\
& -\sin \left(\theta_{i}-\phi_{i}\right)
\end{aligned}
$$

where $x_{G_{i}}$ and $y_{G_{i}}$ are the Cartesian components of the position of point $G_{i}$ (Gosselin and Angeles, 1988) while $h_{1}=\frac{1}{l_{1} l_{3}}$, $h_{2}=\frac{1}{l_{1}}$ and $h_{3}=\frac{1}{l_{3}}$.

\subsection{Link Jacobian matrices}

Since the Principle of Virtual Work is applied to develop the dynamic model of the 3-RRR manipulator, link Jacobian matrices have to be derived. When the end effector is subjected to a virtual displacement, the link Jacobian sub-matrix related to the linear velocity provides the virtual displacement of a point on a link, while the link Jacobian sub-matrix related to angular velocity produces the virtual angular displacement of a link (also referred to as partial velocity and partial angular velocity matrices by some authors, Wu et al., 2009, 2011). Points $G_{i}, B_{i}$ and $P$ are considered as the pivotal points of links $G_{i} B_{i}, B_{i} A_{i}$ and the moving platform, respectively. The link Jacobian sub-matrix related to the angular velocity of link $G_{i} B_{i}$ is written as follows:

$\mathbf{G}_{i 1}=\left[\begin{array}{lll}\frac{-a_{i}}{c_{i}} & \frac{-b_{i}}{c_{i}} & \frac{-d_{i}}{c_{i}}\end{array}\right]$.

The link Jacobian sub-matrix related to the linear velocity of point $G_{i}$ is zero since the velocity of that point is zero. The link Jacobian sub-matrix related to the linear velocity of point $G_{i}$ is thus written as:

$$
\mathbf{H}_{i 1}=\mathbf{0}
$$

The link Jacobian sub-matrix related to the linear velocity of point $B_{i}$ and the link Jacobian sub-matrix related to the angular velocity of link $B_{i} A_{i}$ are obtained from $\mathbf{J}$ (Eq. 18) and from Eq. (14), respectively and are written as follows:

$$
\begin{aligned}
\mathbf{H}_{i 2}= & \frac{l_{1}}{c_{i}}\left[\begin{array}{ccc}
a_{i} \sin \theta_{i} & b_{i} \sin \theta_{i} & d_{i} \sin \theta_{i} \\
-a_{i} \cos \theta_{i} & -b_{i} \cos \theta_{i} & -d_{i} \cos \theta_{i}
\end{array}\right] \\
\mathbf{G}_{i 2}= & {\left[\begin{array}{ll}
\frac{-\sin \beta_{i}}{l_{2}} & \frac{\cos \beta_{i}}{l_{2}}
\end{array}\right]\left(\left[\begin{array}{ll}
\mathbf{e}_{1} & \mathbf{e}_{2}
\end{array}\right]+\mathbf{N R r}_{A_{i}} \mathbf{e}_{3}^{T}\right.} \\
& \left.-\left[\begin{array}{c}
-l_{1} \sin \theta_{i} \\
l_{1} \cos \theta_{i}
\end{array}\right] \mathbf{G}_{i 1}\right)
\end{aligned}
$$

where $\mathbf{e}_{1}=\left[\begin{array}{lll}1 & 0 & 0\end{array}\right]^{T}, \mathbf{e}_{2}=\left[\begin{array}{lll}0 & 1 & 0\end{array}\right]^{T}$ and $\mathbf{e}_{3}=\left[\begin{array}{lll}0 & 0 & 1\end{array}\right]^{T}$.

The link Jacobian sub-matrix related to the angular velocity of the moving platform and the link Jacobian sub-matrix related to the linear velocity of point $P$ are written as follows:

$$
\begin{aligned}
\mathbf{G}_{N} & =\mathbf{e}_{3}^{T} \\
\mathbf{H}_{N} & =\left[\begin{array}{lll}
1 & 0 & 0 \\
0 & 1 & 0
\end{array}\right] .
\end{aligned}
$$

\subsection{Inertial force and inertial moment}

Here, the Newton-Euler formulation is applied to develop the inertial forces and the inertial moments of each moving body about its centre of mass. Then, these inertial forces and moments are calculated about pivotal points (i.e., points $A_{i}, B_{i}$ and $G_{i}$ ). The inertial force and moment of link $G_{i} B_{i}$ about pivotal point $G_{i}$ are written as follows:

$$
\begin{aligned}
\mathbf{F}_{i 1}= & -m_{1}\left(\frac{l_{1}}{2} \ddot{\theta}_{i}\left[-\sin \theta_{i} \cos \theta_{i}\right]^{T}\right. \\
& \left.-\frac{l_{1}}{2} \dot{\theta}_{i}^{2}\left[\cos \theta_{i} \sin \theta_{i}\right]^{T}\right) \\
M_{i 1}= & -\ddot{\theta}_{i} I_{i 1}
\end{aligned}
$$

where $\theta_{i}, \dot{\theta}_{i}$ and $\ddot{\theta}_{i}$ are the angular displacement, angular velocity and angular acceleration of actuator $i$, and $I_{i 1}$ is the moment of inertia of link $G_{i} B_{i}$ about point $G_{i}$.

The influence of internal redundancy appears in the inertial force and moment of the distal links where the moment of inertia and mass centre of the links vary with respect to the position of $m_{s}$. The equations for the inertial force and moment about point $B_{i}$ of the distal links are written as follows:

$$
\begin{aligned}
\mathbf{F}_{i 2} & =-m_{2 \text { tot }}\left(\mathbf{a}_{B_{i}}+r_{i 2} \ddot{\beta}_{i}\left[-\sin \beta_{i} \cos \beta_{i}\right]^{T}\right. \\
& \left.-r_{i 2} \dot{\beta}_{i}^{2}\left[\cos \beta_{i} \sin \beta_{i}\right]^{T}\right)-m_{s} \ddot{s}_{i}\left[\cos \beta_{i} \sin \beta_{i}\right]^{T} \\
& -2 m_{s} \dot{s}_{i} \dot{\beta}_{i}\left[-\sin \left(\beta_{i}\right) \cos \left(\beta_{i}\right)\right]^{T} \\
M_{i 2} & =-\ddot{\beta}_{i} I_{i 2}-m_{2 \text { tot }} r_{i 2}\left[-\sin \beta_{i} \quad \cos \beta_{i}\right] \mathbf{a}_{B_{i}} \\
& -2 m_{s} s_{i} \dot{s}_{i} \dot{\beta}_{i}
\end{aligned}
$$

where $\beta_{i}, \dot{\beta}_{i}$ and $\ddot{\beta}_{i}$ are the displacement, angular velocity and angular acceleration of the passive joints and $m_{2 \text { tot }}$ is the total mass of link $A_{i}^{\prime} A_{i}$, i.e., $m_{2 \text { tot }}=m_{2}+m_{s}$. Also, $\mathbf{a}_{B_{i}}$ describes the linear acceleration of point $B_{i}, r_{i 2}$ is the distance between the centre of mass of link $A_{i}^{\prime} A_{i}$ and point $B_{i}$ while $I_{i 2}$ is the moment of inertia of the distal link with respect to $B_{i}$. The distance from point $B_{i}$ to the barycentre of the redundant mass 
is $s_{i}$ while $\dot{s}_{i}$ and $\ddot{s}_{i}$ describe the velocity and acceleration of $m_{s}$. The position of the centre of mass of the distal link and its moment of inertia vary with respect to the position of the portable mass and are written as follows:

$$
\begin{aligned}
r_{i 2} & =\frac{m_{s} s_{i}+m_{2} r_{G_{2}}}{m_{2}+m_{s}} \\
I_{i 2} & =I_{A_{i}^{\prime} A_{i}}+m_{s}\left(s_{i}\right)^{2}
\end{aligned}
$$

where $r_{G_{2}}$ is the position of the centre of mass of the distal link (excluding $m_{s}$ ) and is equal to zero for the case when $B_{i} A_{i}$ is equal to $B_{i} A_{i}^{\prime}$, and $I_{A_{i}^{\prime} A_{i}}$ is the moment of inertia of link $A_{i}^{\prime} A_{i}$ about its centre of mass (excluding $m_{s}$ ).

The inertial force and moment of the moving platform about point $P$ is written as follows:

$$
\begin{aligned}
\mathbf{F}_{N} & =-m_{n} \mathbf{a}_{P} \\
M_{N} & =-\ddot{\alpha} I_{N}
\end{aligned}
$$

where $\mathbf{a}_{P}$ and $\ddot{\alpha}$ are the linear acceleration of point $P$ and the angular acceleration of the moving platform, respectively while $m_{n}$ and $I_{N}$ represents the mass and the moment of inertia of the moving platform.

\subsection{Dynamic model}

The dynamic equation of the $3-\underline{R} R R$ is written as follows:

$$
\begin{aligned}
\mathbf{J}^{T} \boldsymbol{\tau} & +\sum_{i=1}^{3} \sum_{j=1}^{2}\left[\begin{array}{ll}
\mathbf{H}_{i j}^{T} & \mathbf{G}_{i j}^{T}
\end{array}\right]\left[\begin{array}{ll}
\mathbf{F}_{i j} & \mathbf{M}_{i j}
\end{array}\right]^{T} \\
& +\left[\begin{array}{ll}
\mathbf{H}_{N}^{T} & \mathbf{G}_{N}^{T}
\end{array}\right]\left[\begin{array}{ll}
\mathbf{F}_{N} & \mathbf{M}_{N}
\end{array}\right]^{T}=0
\end{aligned}
$$

where $\mathbf{J}$ is the Jacobian matrix of the manipulator, $\boldsymbol{\tau}$ presents the torque vector, $\mathbf{H}_{i j}$ are the link Jacobian sub-matrices related to velocity and $\mathbf{G}_{i j}$ are the link Jacobian sub-matrices related to the angular velocity of the links, $\mathbf{H}_{N}$ and $\mathbf{G}_{N}$ represent the link Jacobian sub-matrix related to velocity and the link Jacobian sub-matrix related to the angular velocity of the moving platform, $\mathbf{F}_{i j}$ and $\mathbf{M}_{i j}$ are inertial forces and moments of the robot links and $\mathbf{F}_{N}$ and $\mathbf{G}_{N}$ represent the inertial force and moment of the moving platform.

\section{Trajectory optimisation}

When planning a trajectory in the Cartesian space, the displacement, velocity and acceleration of the end effector are known. These can be used to calculate the kinematic properties of all active joints for every point in the trajectory while the dynamic equations can be used to compute the actuator torques. Since the necessary torques to move the end effector are a function of the position, velocity and acceleration of the portable masses, moving the redundant masses (i.e., changing $s_{i}, \dot{s}_{i}$ and $\ddot{s}_{i}$ for $i=1,2,3$ ) will also have a direct effect on the torques at the base-mounted actuators.

Here, variables $s_{i}$ are optimised to minimise the manipulator's total torque at a specific time step within the trajectory.
The optimisation problem is written as follows:

$$
\min _{s_{i}} \sum_{i=1}^{3}\left(\tau_{i}\left(s_{i}\right)-\lambda \bar{\tau}_{i}\right)^{2}
$$

subject to

$$
\begin{aligned}
& -l_{2} \leq s_{i} \leq l_{2} \\
& -\dot{s}_{\max } \leq \dot{s}_{i} \leq \dot{s}_{\max } \\
& -\ddot{s}_{\max } \leq \ddot{s}_{i} \leq \ddot{s}_{\max }
\end{aligned}
$$

where $\tau_{i}$ refers to the optimised torque of actuator $i$ at every time step, $\bar{\tau}_{i}$ is the torque value obtained when a similar manipulator without internal redundancy is used and $\lambda$ is a coefficient between 0 and 1 which makes the objective function flexible on the percentage of the optimised torque value with respect to the torques of the non-redundant manipulator. The optimisation variable (i.e., $s_{i}$ ) is the distance from joint $B_{i}$ to the barycentre of the redundant mass. In Eq. (39), the value of $s_{i}$ has been constrained so as to keep it within track $A_{i}^{\prime} A_{i}$. Also, the rate of change of $s_{i}$ (i.e., $\dot{s}_{i}$ ) is bounded in the positive and negative directions to a maximum absolute value $\dot{s}_{\max }$ (with $\dot{s}_{\max }>0$ ). In addition to that, the rate of change of $\dot{s}_{i}$ (i.e., $\ddot{s}_{i}$ ) is bounded to a maximum absolute value $\ddot{s}_{\max }$. These limits prevent any sudden changes in the motion of the portable masses. The choice of the objective function will be clearer when the results are presented.

During the optimisation procedure, the position of $m_{s i}$, i.e., variable $s_{i}$, changes to minimise the sum of the squared actuator torques within that specific time step. To achieve this, the following steps are followed:

1. Define the reference trajectory (point-to-point): the desired trajectory is planned in Cartesian space and the displacement, velocity and acceleration of the actuators are obtained using the corresponding inverse kinematic solutions.

2. Calculate the torques of the non-redundant manipulator: the torque values of the manipulator without internal redundancy is calculated for the defined reference trajectory.

3. Define the search space: the displacements of the redundant actuators through the trajectory are used as the design variables for the optimisation process.

4. Define the bounds: based on the current position of the portable masses and a user-defined maximum velocity and maximum acceleration of the redundant actuators, the upper and lower bounds of the optimisation variables are calculated.

5. Define the initial condition: the initial position of the portable masses needs to be adjusted as it affects the optimisation results.

6. Define the optimisation stopping criteria: the difference between two consequent optimisation search variables (i.e., displacement of the portable masses) as well 

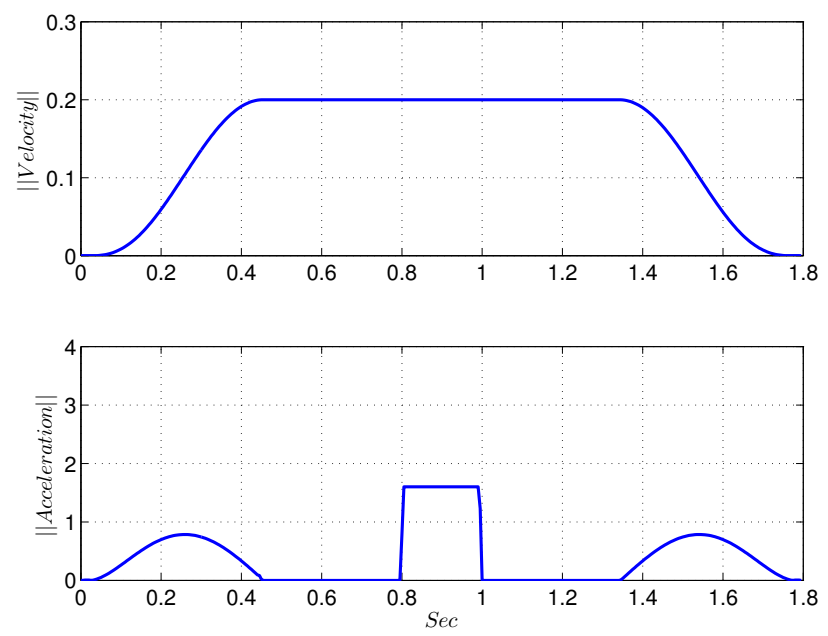

(a) Reference trajectory $r=0.025 \mathrm{~m}$
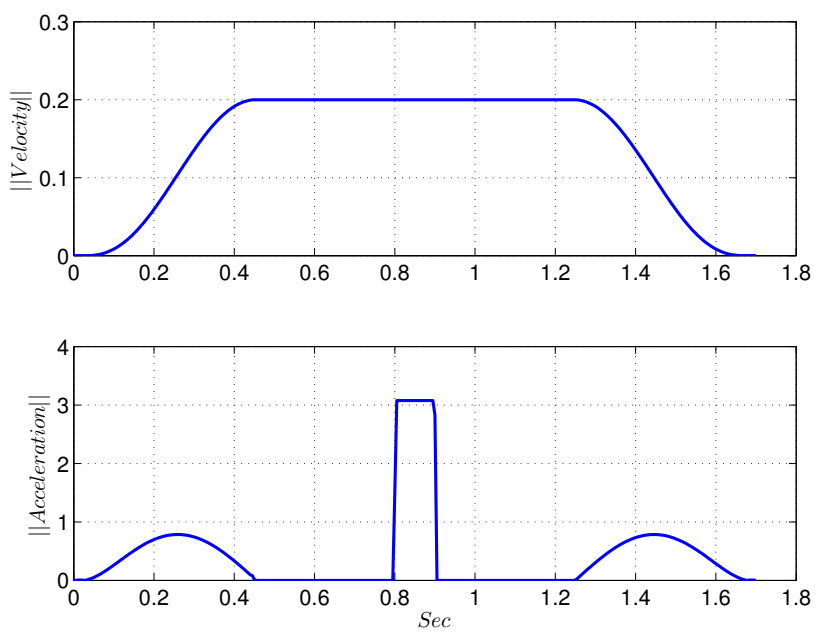

(b) Reference trajectory $r=0.013 \mathrm{~m}$

Figure 3. The norm of the velocity (in $\mathrm{m} \mathrm{s}^{-1}$ ) and the acceleration (in $\mathrm{m} \mathrm{s}^{-2}$ ) of reference trajectories.

as the difference between their objective function values are monitored at every iteration of optimisation. Once they have met the pre-defined user threshold, the optimisation procedure stops.

7. Optimise the position of the portable mass: a nonlinear multi-variable constrained optimisation is conducted to minimise the active-joint torques in Eq. (38).

- The displacement, velocity and acceleration of the base actuators are calculated at every step of the optimisation procedure.

- The current velocity and acceleration of the redundant actuators are calculated using the time history of the redundant actuators.

- The objective function value is determined.

\section{Numerical example}

\subsection{Architectural parameters and analysed trajectory}

The manipulator's architectural parameters for the current example are as follows: all proximal link lengths are set to $1 \mathrm{~m}$ (i.e., $l_{1}=1 \mathrm{~m}$ for all legs). Also, all distal link lengths are set to $1 \mathrm{~m}$ (i.e., $l_{2}=1 \mathrm{~m}$ for all legs) where a track has been attached to every distal link to allow the portable mass to move from $s_{i}=-1$ to $1 \mathrm{~m}$. The base and moving platforms are equilateral triangles inscribed in circles of $1 \mathrm{~m}$ and $0.25 \mathrm{~m}$ in radius, respectively. The mass $m_{1}$ of each of the proximal links is $1 \mathrm{~kg}$ while the distal links have a mass $m_{2}=1 \mathrm{~kg}$ (including the mass of the track) and the end effector has mass $m_{e}=0.5 \mathrm{~kg}$ and $m_{s}=3 \mathrm{~kg}$.

\subsection{Trajectory planning}

The procedure has been studied on two trajectories with rounded corners which have been planned in the Cartesian space. For both trajectories, the end effector moves on a straight line with an initial velocity of $0 \mathrm{~m} \mathrm{~s}^{-1}$ while keeping the end effector with constant orientation. As the tracking velocity reaches a user defined velocity in a specified time $\left(0.2 \mathrm{~m} \mathrm{~s}^{-1}\right.$ in $\left.0.4 \mathrm{~s}\right)$, the end effector tracks the trajectory with a constant velocity. The abrupt acceleration change between $t=0.8$ and $t=1.0 \mathrm{~s}$ occurs when the end-effector enters the rounded corner segment and normal acceleration occurs. The end effector decelerates in $(0.4 \mathrm{~s})$ to come to a stop in the last point of the trajectory. However, the radii of the rounded corners of the trajectories are different.

The trajectory's initial position is $p_{1}=\left[\begin{array}{ll}1 & 0.4\end{array}\right]^{T}$. Also, the radii of the round corners are $r=0.025 \mathrm{~m}$ and $r=0.013 \mathrm{~m}$. Each trajectory starts from point $p_{1}$ and goes in the positive $Y$ direction. Once the end effector moves $0.07 \mathrm{~m}$ in the $Y$ direction, the rounded corner commences (the rounded corner is a quarter of a circle). Thereafter, the end effector travels $0.07 \mathrm{~m}$ in the negative $x$ direction. The norm of the Cartesian velocity and the acceleration of the end effector is presented in Fig. 3. Since the radii of the rounded corners are different, the total length of the trajectories are not the same.

The optimisation problem was implemented in Matlab. The function fmincon was used to perform the constrained local optimisation in Eqs. (38) to (40). More particularly, the Sequential Quadratic Programming (SQP) with Hessian update option within fmincon was used. The SQP method is an alternative approach for handling inequality constraints in non-linear programming where SQP finds the minimum of a sequence of quadratic programming sub-problems. The objective function is estimated with a quadratic function and 

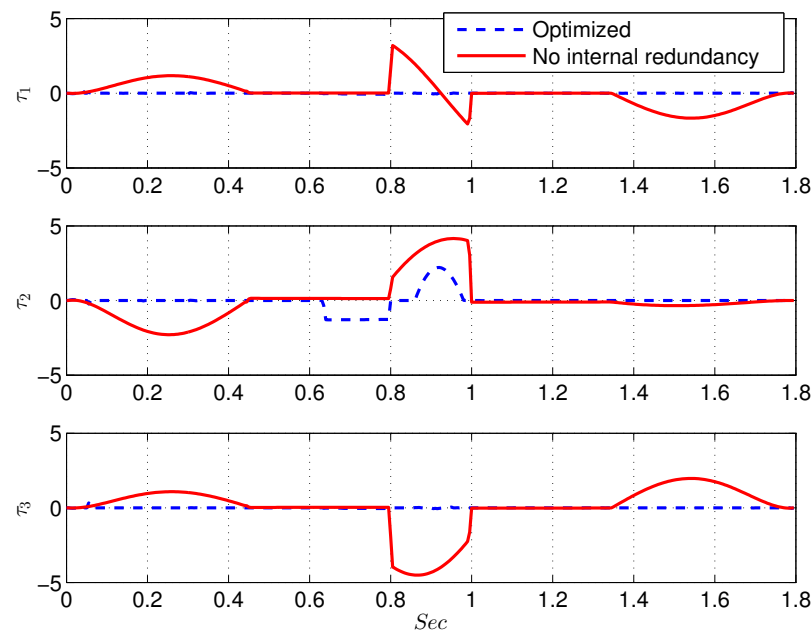

(a) Base joint torque for $r=0.025 \mathrm{~m}$

Figure 4. The torques of the ground actuators (in $\mathrm{Nm}$ ) for $\lambda=0$.

is minimised subject to the linearised constraints. In this method, the Hessian of the Lagrangian function is estimated at every iteration using a quasi-Newton update method. This approximation is used to create a quadratic programming sub-problem and its solution is applied to generate a search direction for the line search procedure (Fletcher, 1987).

In the current numerical example, the velocity of the portable masses is allowed to vary in the range between $-1 \mathrm{~m} \mathrm{~s}^{-1}$ and $+1 \mathrm{~m} \mathrm{~s}^{-1}$. The maximum absolute value of the acceleration of the portable masses is considered as $7 \mathrm{~m} \mathrm{~s}^{-2}$ and $m_{s_{i}}=3 \mathrm{~kg}$ for $i=1,2,3$.

\section{Results and discussion}

Figure 4a illustrates the comparison between the torque values obtained from the optimisation routine and the manipulator without internal redundancy (i.e., $m_{s_{i}}=I_{B_{i} A_{i}^{\prime}}=0$ ) for the trajectory with $r=0.025 \mathrm{~m}$ as the radius of the rounded corner and $\lambda=0$. As shown in Fig. 4a, the manipulator with internal redundancy can follow the reference trajectory with significantly lower torques (approximately $10^{-1} \mathrm{Nm}$ ) in both the accelerating and decelerating phases as well as the rounded corner area. However, the optimised torque for joint two is greater than the non-optimised one at $t=0.65 \mathrm{~s}$. As can be seen in Fig. 6a, the acceleration of the portable mass is zero at $t=0.65 \mathrm{~s}$ which means the velocity of the portable mass meets the limits. Consequently, the effect of $\ddot{s_{1}}$ is eliminated from the dynamic equation at that instant. Also, the optimised torque for joint two at $t=0.9 \mathrm{~s}$ is slightly greater than the optimised torque of actuators one and three at the same time instant. As it is shown in Fig. 6a, the acceleration of the second portable mass meets the limit at $t=0.9 \mathrm{~s}$. Consequently, the inertial force that is produced due to the
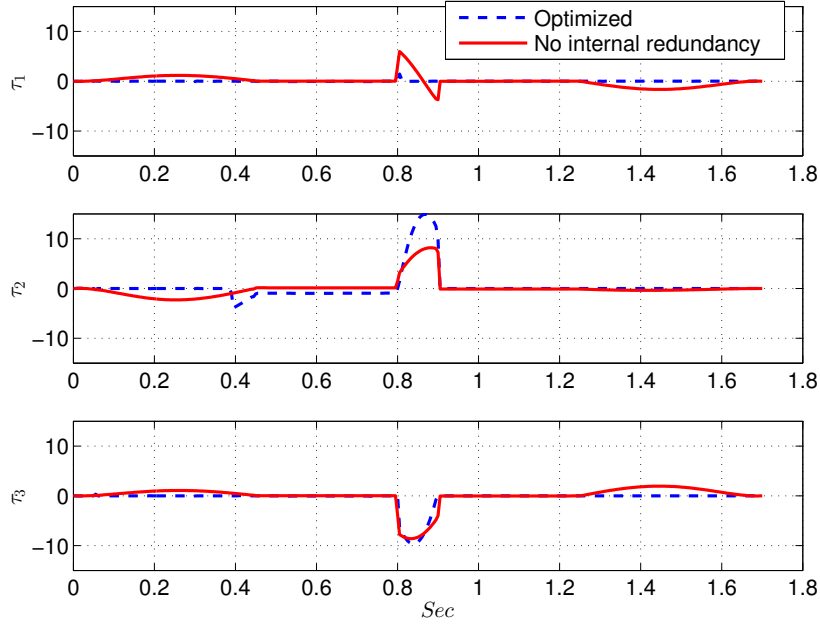

(b) Base joint torque for $r=0.013 \mathrm{~m}$

motion of the portable mass remains constant as well as its improving effect on the torque.

Figure $4 \mathrm{~b}$ presents the result of the optimised torques against non-optimised ones for the trajectory with a smaller rounded corner radius (i.e., $r=0.013 \mathrm{~m}$ ). As can be seen in the torque plot of joints 2 and 3 , the optimised value of the torques are greater than the non-optimised value when the end effector goes through the rounded corner. This is due to the acceleration of the portable mass (i.e., $\ddot{s}_{1}$ ) which meets the pre-defined threshold (see Fig. 6b). At this point, the accelerations of the portable masses remain constant as well as their effect on the inertial force of the distal link. Similar to the results for the trajectory with $r=0.025 \mathrm{~m}$, the velocity of the portable mass two meets the limit at $t=0.4 \mathrm{~s}$ and the corresponding acceleration drops to zero. It has been noticed that the optimised value of the torque of joint 2 and 3 will be less than non-optimised one if the limit of the acceleration of the portable mass is increased to $12 \mathrm{~m} \mathrm{~s}^{-2}$. Also, there is a small jump at the optimised torque value of joint 1 at $t=0.8 \mathrm{~s}$. This is due to the acceleration of the portable mass that meets the limit.

Figure 5a shows the result of optimisation of the torques for the trajectory with $r=0.025 \mathrm{~m}$ wile $\lambda=0.5$. Since the optimised torque values need to be as small as half of the torque values of non-redundant manipulator, the portable masses need to produce smaller inertial forces and moments in comparison with the scenario with $\lambda=0$ (Fig. 4a). Consequently, the portable masses move with smaller velocity and acceleration (Fig. 6c) which prevents them from meeting the limits. As it is seen in Fig. 6c, all portable masses move with relatively smaller acceleration in comparison with Fig. 6a. Also, the second portable mass does not meet the velocity limit at $t=0.65 \mathrm{~s}$. The displacement of the portable masses is shown 

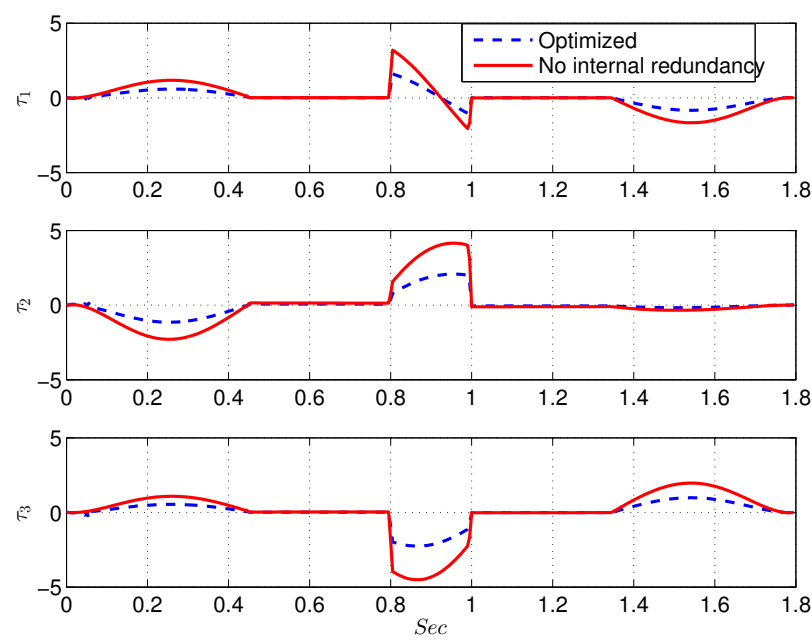

(a) Base joint torque
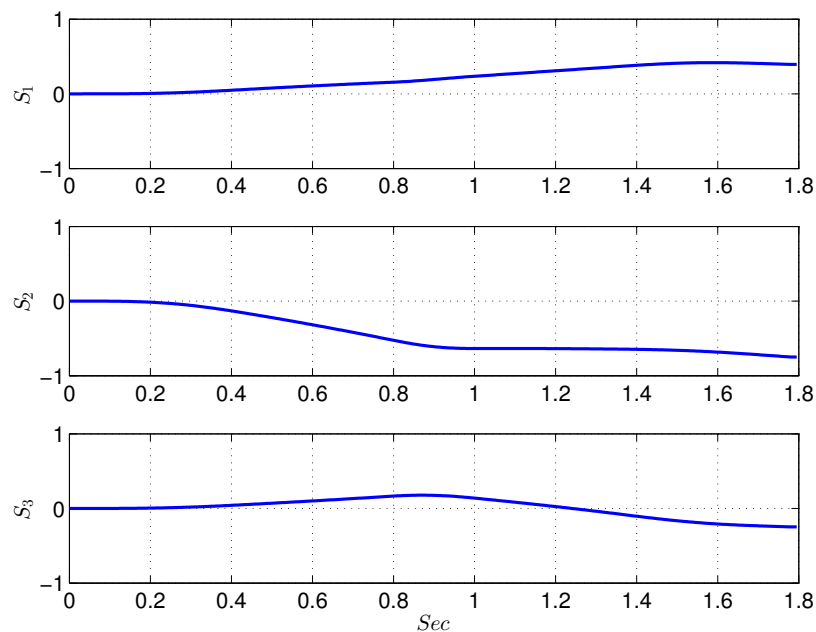

(b) Displacement of portable masses

Figure 5. The torques of the ground actuators (in Nm) and displacement of the portable masses (in $\mathrm{m}$ ) for $r=0.025 \mathrm{~m}$ and $\lambda=0.5$.

in Fig. $5 \mathrm{~b}$ where they are initial at 0 and are allowed to move between -1 and 1 .

The torque values of the joints are relatively small in all cases when the end effector moves with a constant velocity. When the acceleration of the end effector is zero, the inertial forces and moments of the links decrease. In addition to that, the inertial force of the end effector will turn to zero.

\section{Conclusions}

The dynamic model of a 3-RRR planar parallel manipulator involving a portable mass on the distal links is developed. The total of the squared actuators torques is investigated. An optimisation algorithm is implemented to find the optimal position of the portable masses while the end effector undergoes an arbitrary trajectory with a rounded corner.

The concept was tested on two trajectories with different rounded corners using the same Cartesian velocity. The results of the conducted tests suggest that the motion of the portable masses can improve (i.e., reduce) the ground actuator torques for both accelerating and decelerating sections. Also, the base actuator torques improve when the end effector tracks the rounded corner with $r=0.025 \mathrm{~m}$. However, the optimised torques are greater than the the nonoptimised ones around the rounded corner for the trajectory with $r=0.013 \mathrm{~m}$. Since the trajectory with sharper corner imposes greater torque values on the ground joints, the motion of the portable masses need to generate greater inertial forces and moments on the distal links to improve the torque values at rounded corner. However, the changes in inertial forces and moments of the distal links are limited due the limits that have been defined for the velocity and acceleration of the portable masses.
The objective function is flexible to determine the percentage of improvement of the optimised torques with respect to the torque values of the same manipulator without internal redundancy. As greater improvement of the torques requires higher limits of the velocity and the acceleration for portable masses, the objective function can be adjusted to keep the optimisation variables away from the limits.

The obtained simulation results suggest that if a manipulator can not follow a trajectory with a rounded corner due to the torque limits of the ground joints, it will be feasible through application of internal redundancy (without altering the ground actuators). This is possible as the dynamic forces required to perform the more demanding trajectories are shared by both the base actuators as well as the additional actuators on the distal links.

There are a few parameters that affect the the simulation such as Cartesian velocity of the end effector, the radius of the rounded corner and the allowed limits of the velocity and acceleration of the portable masses. For instance, having a relatively large end effector velocity demands greater torque values at the ground joints. Consequently, the portable masses need to generate greater forces and moments on the distal links which is proportional to the limits of the velocity and acceleration of the portable masses. Moreover, due the aforementioned force sharing effect, the balance between the contribution of the two sets of actuators to the specific task needs to be carefully considered (e.g., using an objective function that considers both sets of actuators).

As future work, it is suggested to look at the trajectory globally rather than point-to-point motion planning. In that case, the position of the portable masses can be adjusted with respect to the any up-coming critical situation (i.e., rounded corner). 

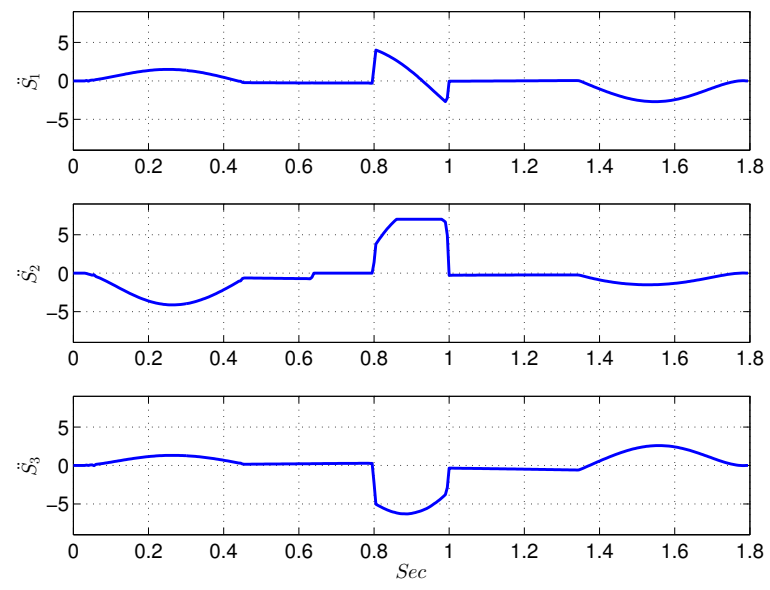

(a) $r=0.025$ (m) and $\lambda=0$
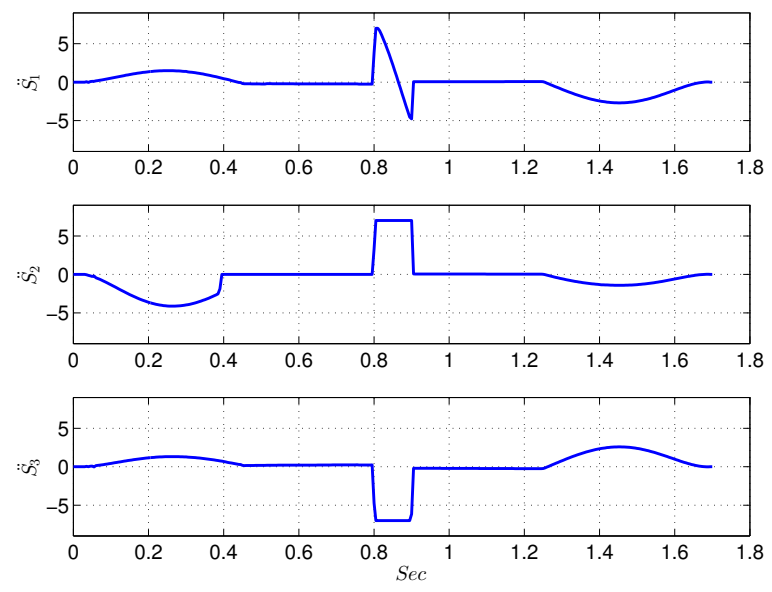

(b) $r=0.013(\mathrm{~m})$ and $\lambda=0$
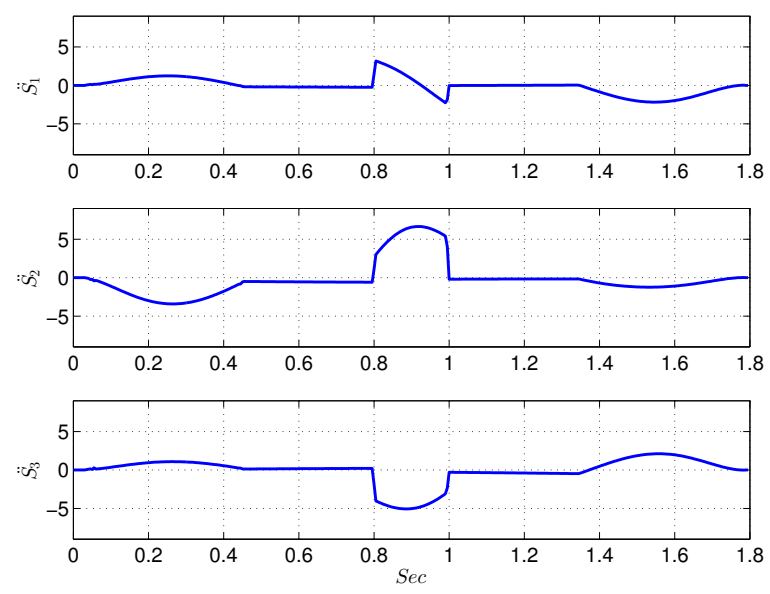

(c) $r=0.025(\mathrm{~m})$ and $\lambda=0.5$

Figure 6. The acceleration of the portable masses $\left(\right.$ in $\mathrm{m} \mathrm{s}^{-2}$ ).
Acknowledgement. The authors acknowledge the financial support from the Natural Science and Engineering Research council of Canada through their Discovery Grant program.

Edited by: A. Müller

Reviewed by: two anonymous referees

\section{References}

Boudreau, R. and Nokleby, S.: Force Optimisation of kinematicallyredundant planar parallel manipulators following a desired trajectory, Mech. Mach. Theory, 56, 138-155, 2012.

Cha, S., Lasky, T. A., and Velinsky, S. A.: Kinematically-Redundant Variations of the 3-RRR Mechanism and Local OptimizationBased Singularity Avoidance, Mechanism Based Design of Structures and Machines, 35, 15-38, 2007.

Cheng, H., Yiu, Y.-K., and Li, Z.: Dynamics and Control of Redundantly Actuated Parallel Manipulators, IEEE/ASME Transactions on Mechatronics, 8, 483-491, doi:10.1109/TMECH.2003.820006, 2003.

Cheng, H., Liu, G. F., Yiu, Y. K., Xiong, Z. H., and Li, Z.: Advantages and dynamics of parallel manipulators with redundant actuation, in: Proceedings of the IEEE/RSJ International Conference on Intelligent Robots and Systems, 171-176, Maui, USA, 2011.

Ebrahimi, I., Carretero, J. A., and Boudreau, R.: Kinematic analysis and path planning of a new kinematically redundant planar parallel manipulator, Robotica, 26, 405-413, 2008.

Firmani, F. and Podhorodeski, R. P.: Force-unconstrained poses for a redundantly actuated planar parallel manipulator, Mech. Mach. Theory, 39, 459-476, 2004.

Firmani, F., Zibil, A., Nokleby, S. B., and Podhorodeski, R. P.: Force-Moment Capabilities of Revolute-Jointed Planar Parallel Manipulators with Additional Actuated Branches, T. Can. Soc. Mech. Eng., 31, 469-481, http://www.tcsme.org/Vol31-No4. html, 2007.

Fletcher, R.: Practical Methods of Optimization, John Wiley and Sons, 1987.

Garg, V., Carretero, J. A., and Nokleby, S. B.: A New Method to Determine the Force and Moment Workspaces of Actuation Redundant Spatial Parallel Manipulators, Journal of Mechanisms and Robotics, 1, 1-8, doi:10.1115/1.3147184, 2009.

Gosselin, C. and Angeles, J.: The optimum kinematic design of a planar three-degree-of-freedom parallel manipulator, Journal of Mechanism, Transmissions, and Automation in Design, 110, 35 41, 1988.

Lee, S. and Kim, S.: Kinematic analysis of generalized parallel manipulator systems, in: Proceedings of the IEEE Conference on Decision and Control, 2, 1097-1102, 1993.

Merlet, J.-P.: Redundant parallel manipulators, Lab. Robotics Automat., 8, 17-24, 1996.

Nokleby, S. B., Fisher, R., Podhorodeski, R. P., and Firmani, F.: Wrench capabilities of redundantly-actuated parallel manipulators, Mech. Mach. Theory, 40, 578-599, doi:10.1016/j.mechmachtheory.2004.10.005, 2005.

Ruggiu, M. and Carretero, J. A.: Kinematic Analysis of the 3-PRPR Redundant Planar Parallel Manipulator, in: Proceedings of the 2009 CCToMM Symposium on Mechanisms, Machines, and Mechatronics, Québec, Canada, 2009. 
Ruggiu, M. and Carretero, J. A.: Actuation strategy based on the acceleration model for the 3-PRPR redundant planar parallel manipulator, in: Advances in Robot Kinematics: Analysis and Design, edited by: Lenarčič, J. and Stanisic, M. M., Springer, PiranPortorož, Slovenia, 2010.

Vukobratović, M., Potkonjak, V., and Matijević, V.: Internal redundancy - the way to improve robot dynamics and control performances, J. Intell. Robot. Syst., 27, 31-66, 2000.

Wang, J. and Gosselin, C. M.: Kinematic Analysis and Design Of Kinematically Redundant Parallel Mechanisms, J. Mech. Design, 126, 109-118, 2004.
Wu, J., Wang, J., Wang, L., and Tiemin, L.: Dynamics and control of a planar 3-DOF parallel manipulator with actuation redundancy, Mech. Mach. Theory, 44, 835-849, 2009.

Wu, J., Wang, J., and You, Z.: A comparison study on the dynamics of planar 3-DOF 4-RRR, 3-RRR and 2-RRR parallel manipulators, Journal of Robotics and Computer Integrated Manufacturing, 27, 150-156, 2011.

Zanganeh, K. E. and Angeles, J.: Instantaneous kinematics and design of a novel redundant parallel manipulator, in: Proceedings of IEEE Conference on Robotics and Automation, 3043-3048, San Diego, USA, 1994. 